\title{
Insulin sensitivity in juvenile and adult Large White pigs of low and high birthweight
}

\author{
K. R. Poore ${ }^{1,2}$ - A. L. Fowden 1 \\ ${ }^{1}$ Department of Physiology, University of Cambridge, Cambridge, UK \\ ${ }^{2}$ Centre for Fetal Origins of Adult Disease, University of Southampton, Princess Anne Hospital, Southampton, UK
}

\begin{abstract}
Aims/hypothesis. We have previously demonstrated poor glucose tolerance in adult pigs of naturally occurring low birthweight. The aim of this study was to examine sensitivity to insulin in juvenile (3-monthold) and adult (12-month-old) pigs of low and high birthweight.

Methods. Low $(<1.47 \mathrm{~kg})$ and high $(>1.53 \mathrm{~kg})$ birthweight piglets from 15 litters were studied at 3 $(n=47)$ and $12(n=17)$ months of age. At each age the selected pigs were tranquilised and catheters were inserted into the dorsal aorta and caudal vena cava under general anaesthesia. After recovery, insulin sensitivity was measured as the glucose decrement $\left(\mathrm{mmol} \cdot \mathrm{l}^{-1} \cdot \mathrm{min}^{-1}\right)$ during the first $10 \mathrm{~min}$ after an intravenous insulin bolus $(0.5 \mathrm{IU} / \mathrm{kg})$. Data (means \pm SEM) were analysed by the Student's $t$ test, ANOVA and linear regression.
\end{abstract}

Results. The body weight of low birthweight female, but not male, pigs remained smaller than that of high birthweight pigs at 3 and 12 months of age. At 3 months, thinness at birth and rapid catch-up growth in the first month of life were associated with increased insulin sensitivity in males. In females thinness at 3 months was associated with reduced sensitivity to insulin. At 12 months, early postnatal catch-up growth was associated with insulin resistance, irrespective of sex, when all data were combined.

Conclusions/interpretation. The glucose intolerance previously observed in young adult pigs of low birthweight is probably due to insulin resistance. Early catch-up growth in low birthweight pigs was the clearest predictor of adult insulin resistance. [Diabetologia (2004) 47:340-348]

Keywords Low birthweight $\cdot$ Postnatal catch-up growth $\cdot$ Insulin sensitivity $\cdot$ Pigs
In man, low birthweight and small size at birth are associated with an increased risk of cardiovascular disease, glucose intolerance and non-insulin dependent Type 2 diabetes in adult life $[1,2,3]$. These associa-

Received: 5 May 2003 / Revised: 13 October 2003

Published online: 14 January 2004

(C) Springer-Verlag 2004

K. R. Poore ( $\bullet)$

Centre for Fetal Origins of Adult Disease,

University of Southampton, Princess Anne Hospital,

Level F (MP 887), Coxford Road, Southampton,

SO16 5YA, UK

E-mail: kpoore@soton.ac.uk

Abbreviations: AC, abdominal circumference tions have been linked to poor nutrition during pregnancy and early postnatal life and have led to the 'thrifty phenotype' hypothesis [4]. This hypothesis proposes that when nutritional conditions in utero are suboptimal, metabolic and endocrine adaptations occur in the fetus and slow intrauterine growth, with beneficial consequences for fetal survival, but detrimental effects on postnatal glucose handling once nutrition is restored to normal after birth.

The thrifty hypothesis has been tested experimentally in several species using various methods to impair fetal growth, including low protein or global restriction of maternal diet, placental insufficiency and glucocorticoid administration $[5,6,7,8]$. These studies support the hypothesis and confirm that impaired intrauterine development can have consequences for 
glucose metabolism long after birth. In addition, spontaneously occurring growth retardation in polytocous species such as pigs show that low birthweight is associated with disturbed glucose metabolism in later life [9].

In normally fed pigs, there is a natural two- to three-fold variation in birthweight amongst littermates due to differences in placental size and functional capacity [10]. Piglets of low birthweight are frequently thin and of disproportionate body shape, both of which are good indices of growth retardation during late gestation [10]. Recent studies have shown that low-birthweight piglets have abnormal cardiovascular function as juveniles and poor glucose tolerance as adults $[9,11]$. The cause of adult glucose intolerance was not identified, but did not appear to involve insulin deficiency. The aim of our study was, therefore, to examine sensitivity to exogenous insulin, as an index of insulin resistance, in juvenile (3-month-old) and adult (12-month-old) pigs of low and high birthweight. Insulin sensitivity was measured as the fall in plasma glucose after an intravenous injection of a bolus dose of porcine insulin. A preliminary account of some of these data has already been published [12].

\section{Materials and methods}

Animals. Purebred Large White pigs were obtained from sows (Department of Clinical Veterinary Medicine, University of Cambridge, Cambridge, UK) allowed to farrow normally at term (115 \pm 2 days). Fifteen litters from nine sows were used in this study (average number of piglets per litter: 11.3 \pm 0.9 ). Sows were fed a standard diet $(15 \%$ protein; $12.6 \mathrm{MJ} / \mathrm{kg}$ digestible energy; ABN, Woodstone, Peterborough, UK) at least 4 weeks prior to conception ( $2 \mathrm{~kg}$ per day) and during gestation and lactation (2.5-3 kg per day) and according to standard guidelines [13]. Free access to water was provided. Piglets were provided with straw bedding and infrared heat lamps from birth until weaning at 4 to 5 weeks of age. Weaner piglets were housed in groups and had free access to a standard pig creep diet (20\% protein; H \& C Beart, Brighton Mill, Kings Lynn, UK) until the first studies were done at 3 months of age. From this time, the pigs were housed individually adjacent to their siblings and fed twice a day according to their size $(0.5 \mathrm{~kg}$ per $30 \mathrm{~kg}$ body weight). On completion of all studies at 3 months of age, the pigs were returned to group housing (free access to $20 \%$ protein pig creep) until prior to puberty. At 4 to 5 months of age, they were again housed individually and fed on the adult $15 \%$ protein diet for the remainder of the study.

At birth, all piglets in each litter were weighed and the following morphometric measurements made: head length (snout to between the ears), crown-rump length (between the ears to base of tail) and abdominal circumference (AC). The average birthweight of all piglets born for this study was $1.50 \pm 0.02 \mathrm{~kg}$ $(n=170)$ and the $95 \% \mathrm{CI}$ of the mean was 1.47 to $1.53 \mathrm{~kg}$. After measurement of birthweight, piglets were assigned to one of two groups: low-birthweight piglets (less than the $95 \% \mathrm{CI}$ of the mean birthweight, i.e. $<1.47 \mathrm{~kg}$ at birth); and high-birthweight piglets (higher than the $95 \%$ CI of the mean birthweight, i.e. $>1.53 \mathrm{~kg}$ at birth). The range of birthweights in the low-birthweight group was $0.80-1.40 \mathrm{~kg}(n=22)$. In the highbirthweight group the range was $1.65-2.40 \mathrm{~kg}(n=25)$. The fol- lowing numbers of each sex were selected: low-birthweight females, $n=15$; low-birthweight males, $n=7$; high-birthweight females, $n=13$; high-birthweight males, $n=12$. The selected pigs were weighed and measured again at 1, 3 and 12 months of age. It was not possible to obtain all data from all animals: the number of observations for each experimental data set is indicated in the relevant Figure legend or table footnote.

At 9 to 10 weeks of age, before the morning feed, the selected pigs were tranquilised with azaperone $(5 \mathrm{mg} / \mathrm{kg}$ intramuscularly for pigs above $20 \mathrm{~kg}$; Janssen Pharmaceuticals, Oxford, UK) or diazepam (2 $\mathrm{mg} / \mathrm{kg}$ intramuscularly for pigs less than $20 \mathrm{~kg}$; Phoenix Pharmaceuticals, Gloucester, UK), each in combination with ketamine $(10 \mathrm{mg} / \mathrm{kg}$ intramuscularly; Fort Dodge Animal Health, Southampton, UK), and anaesthetised with halothane ( 3 to $6 \%$ in $\mathrm{O}_{2}$ ). Catheters were inserted into the dorsal aorta and vena cava via the femoral vessels and were exteriorised via a small incision on the animal's back. Pigs were kept in protective coats made of elastic tubing (Tubigrip, Seton Healthcare, Oldham, UK) to protect the catheters. Antibiotic treatment was given on the day of surgery [procaine benzylpenicillin, $15 \mathrm{mg} / \mathrm{kg}$, Mycofarm, Cambridge, UK; and Duphatrim (trimethoprim, $2.5 \mathrm{mg} / \mathrm{kg}$, with sulfadiazine, $12.5 \mathrm{mg} / \mathrm{kg}$, Fort Dodge Animal Health); all injected intramuscularly], for 3 days after surgery and every 2 to 3 days thereafter (Duphatrim alone, i.v.). The normal feeding regime was restored immediately after recovery from surgery.

Seventeen pigs had their catheters and protective coats removed on completion of the experiments at 3 months of age and were then studied again at 10 to 12 months of age (lowbirthweight females, $n=5$; low-birthweight males, $n=4$; highbirthweight females, $n=4$; high-birthweight males, $n=4$ ). Femoral artery and vein catheters were inserted in the previously unoperated leg under general anaesthesia (sodium pentobarbitone, $20 \mathrm{mg} / \mathrm{kg}$ i.v.; Rhône Mérieux, Harlow, UK) after tranquilisation with azaperone ( $5 \mathrm{mg} / \mathrm{kg}$ intramuscularly).

All procedures were carried out in accordance with the regulations of the UK Home Office Animals (Scientific Procedures) Act, 1986.

Experimental protocol. After allowing at least 2 days for recovery from surgery, an insulin challenge was performed, following an overnight fast. Porcine insulin $(0.5 \mathrm{IU} / \mathrm{kg}$ body weight; Novo Nordisk Pharmaceuticals, Crawley, UK) was given as a rapid intravenous bolus. Arterial blood samples $(2 \mathrm{ml})$ were collected into chilled EDTA tubes for analysis of glucose concentrations at $-30 \mathrm{~min},-15 \mathrm{~min}$ and immediately before insulin was given, and at 5, 10, 15, 30, 45, 60, 90 and $120 \mathrm{~min}$ after the insulin bolus. All blood samples were centrifuged immediately for $5 \mathrm{~min}$ at $4^{\circ} \mathrm{C}$ and, after measurement of plasma glucose concentrations, the plasma was stored at $-20^{\circ} \mathrm{C}$. For each insulin challenge experiment, the rate of glucose fall from the mean concentrations before the insulin injection ( -30 to $0 \mathrm{~min}$ ) to the first $10 \mathrm{~min}$ after insulin administration was calculated $\left(\mathrm{mmol} \cdot \mathrm{l}^{-1} \cdot \mathrm{min}^{-1}\right.$; glucose decrement).

Biochemical analyses. Plasma glucose concentrations were measured using an automated analyser (Yellow Springs 2300 Stat Plus Glucose/Lactate analyser; YSI, Farnborough, UK). Plasma insulin concentrations were measured using a commercially available radioimmunoassay kit (INSIK-5; Diasorin, Wokingham, UK). The inter-assay and intra-assay coefficients of variation for the insulin assay were $10 \%$ and $8 \%$ respectively. Basal glucose and insulin concentrations for each animal were each given by the means of two to three fasted samples taken on separate experimental days.

Statistics. All results are expressed as means \pm SEM. The relationships between two factors, the measured dependent vari- 
Table 1. Body weights and length to weight ratios at birth and at 3 and 12 months of age in low- and high-birthweight (BW) male and female pigs

\begin{tabular}{|c|c|c|c|c|c|c|}
\hline & \multicolumn{2}{|l|}{ All } & \multicolumn{2}{|l|}{ Males } & \multicolumn{2}{|l|}{ Females } \\
\hline & Low BW & High BW & Low BW & High BW & Low BW & High BW \\
\hline BW (kg) & $1.13 \pm 0.04^{\mathrm{d}}$ & $1.90 \pm 0.04$ & $1.18 \pm 0.05^{\mathrm{d}}$ & $1.93 \pm 0.05$ & $1.10 \pm 0.05^{\mathrm{d}}$ & $1.86 \pm 0.05$ \\
\hline PI $\left(\mathrm{kg} / \mathrm{CRL}^{3}\right)$ & $70.3 \pm 1.6$ & $72.3 \pm 2.5$ & $72.63 \pm 4.28$ & $73.63 \pm 3.71$ & $68.45 \pm 1.30$ & $73.28 \pm 3.41$ \\
\hline BMI (kg/CRL $\left.{ }^{2}\right)$ & $17.7 \pm 0.3^{\mathrm{d}}$ & $21.4 \pm 0.5$ & $18.26 \pm 0.67^{b}$ & $21.83 \pm 0.79$ & $17.30 \pm 0.31^{\mathrm{d}}$ & $21.45 \pm 0.68$ \\
\hline $\mathrm{CW}(\mathrm{kg})$ & $23.5 \pm 1.9^{\mathrm{d}}$ & $37.2 \pm 2.5$ & $25.57 \pm 4.62$ & $36.07 \pm 2.97$ & $22.60 \pm 1.84^{\mathrm{c}}$ & $38.23 \pm 4.11$ \\
\hline PI $\left(\mathrm{kg} / \mathrm{CRL}^{3}\right)$ & $55.6 \pm 3.2$ & $59.6 \pm 1.6$ & $53.08 \pm 9.01$ & $61.82 \pm 2.02$ & $57.02 \pm 3.63$ & $59.64 \pm 2.70$ \\
\hline BMI (kg/CRL $\left.{ }^{2}\right)$ & $40.9 \pm 2.4^{a}$ & $51.6 \pm 2.7$ & $39.20 \pm 12.33$ & $49.39 \pm 3.79$ & $42.69 \pm 1.86$ & $51.60 \pm 4.25$ \\
\hline \multicolumn{7}{|c|}{ At 12 months of age: } \\
\hline $\mathrm{CW}(\mathrm{kg})$ & $152.8 \pm 8.1$ & $169.4 \pm 6.0$ & $168.8 \pm 13.9$ & $176.3 \pm 9.9$ & $140.0 \pm 5.5^{\mathrm{a}}$ & $162.5 \pm 6.3$ \\
\hline
\end{tabular}

Values are means \pm SEM. Low BW vs high BW across all or within each sex (unpaired $t$ test): ${ }^{\mathrm{a}} p<0.05,{ }^{\mathrm{b}} p<0.01,{ }^{\mathrm{c}} p<0.005$, d $p<0.001$

Numbers per group at 3 months: low-BW males, $n=7$; high-

$\mathrm{BW}$ males, $n=12$; low-BW females, $n=15$; high-BW females $n=13$. Numbers per group at 12 months: low-BW males, $n=4$; high-BW males, $n=4$; low-BW females, $n=5$; high-BW females $n=4$. BW, birthweight; PI, ponderal index; CW, current weight

Table 2. Morphometric measurement ratios at birth and at 3 and 12 months of age in low- and high-birthweight (BW) male and female pigs

\begin{tabular}{|c|c|c|c|c|c|c|}
\hline & \multicolumn{2}{|l|}{ All } & \multicolumn{2}{|l|}{ Males } & \multicolumn{2}{|l|}{ Females } \\
\hline & Low BW & High BW & Low BW & High BW & Low BW & High BW \\
\hline Head length:BW $(\mathrm{cm} / \mathrm{kg})$ & $8.78 \pm 0.36^{\mathrm{d}}$ & $5.73 \pm 0.14$ & $8.21 \pm 0.63^{d}$ & $5.67 \pm 0.23$ & $8.97 \pm 0.44^{\mathrm{d}}$ & $5.65 \pm 0.17$ \\
\hline Head length:CRL & $0.385 \pm 0.012$ & $0.365 \pm 0.009$ & $0.378 \pm 0.029$ & $0.366 \pm 0.013$ & $0.385 \pm 0.011$ & $0.362 \pm 0.013$ \\
\hline Head length:AC & $0.449 \pm 0.017^{\mathrm{a}}$ & $0.403 \pm 0.010$ & $0.433 \pm 0.042$ & $0.416 \pm 0.013$ & $0.453 \pm 0.017^{\mathrm{a}}$ & $0.393 \pm 0.014$ \\
\hline Head length:CW $(\mathrm{cm} / \mathrm{kg})$ & $0.99 \pm 0.09^{\mathrm{a}}$ & $0.63 \pm 0.08$ & $1.17 \pm 0.53$ & $0.68 \pm 0.07$ & $0.91 \pm 0.05$ & $0.63 \pm 0.15$ \\
\hline Head length:CRL & $0.288 \pm 0.001$ & $0.288 \pm 0.009$ & $0.278 \pm 0.001$ & $0.282 \pm 0.009$ & $0.289 \pm 0.014$ & $0.288 \pm 0.016$ \\
\hline Head length:AC & $0.339 \pm 0.014^{\mathrm{a}}$ & $0.303 \pm 0.010$ & $0.325 \pm 0.015$ & $0.298 \pm 0.013$ & $0.338 \pm 0.018$ & $0.303 \pm 0.016$ \\
\hline \multicolumn{7}{|l|}{ At 12 months of age: } \\
\hline Head length:CW $(\mathrm{cm} / \mathrm{kg})$ & $0.223 \pm 0.016$ & $0.260 \pm 0.035$ & $0.23 \pm 0.03$ & $0.21 \pm 0.01$ & $0.218 \pm 0.023$ & $0.295 \pm 0.050$ \\
\hline
\end{tabular}

Values are means \pm SEM. Low BW vs high BW across all or within each sex (unpaired $t$ test): a $p<0.05$, d $p<0.001$

Numbers per group at 3 months: low-BW males, $n=7$; highBW males, $n=12$; low-BW females, $n=15$; high-BW females

ables versus particular independent variables, were tested using separate correlation analyses. The effects of birthweight group and time were tested using multifactorial analyses of variance. Unpaired Student's $t$ tests were used to identify differences between two factors. Statistical analyses were done using SigmaStat Statistical Software version 2.0 (SPSS, Chicago, Ill., USA) and for all tests statistical significance was accepted at a $p$ value of less than 0.05 . $n=13$. Numbers per group at 12 months: low-BW males, $n=4$; high-BW males, $n=4$; low-BW females, $n=5$; high-BW females $n=4$. BW, birthweight; CRL, crown rump length; AC, abdominal circumference; $\mathrm{CW}$, current weight

\section{Results}

Postnatal growth. There were significant $(p<0.01)$ differences in body weight, BMI and the ratio of head length to body weight between low- and high-birthweight pigs at birth, and these differences were similar in males and females (Table 1 and Table 2). Overall, the current weight at a given time was related to birthweight at 3 but not at 12 months of age 

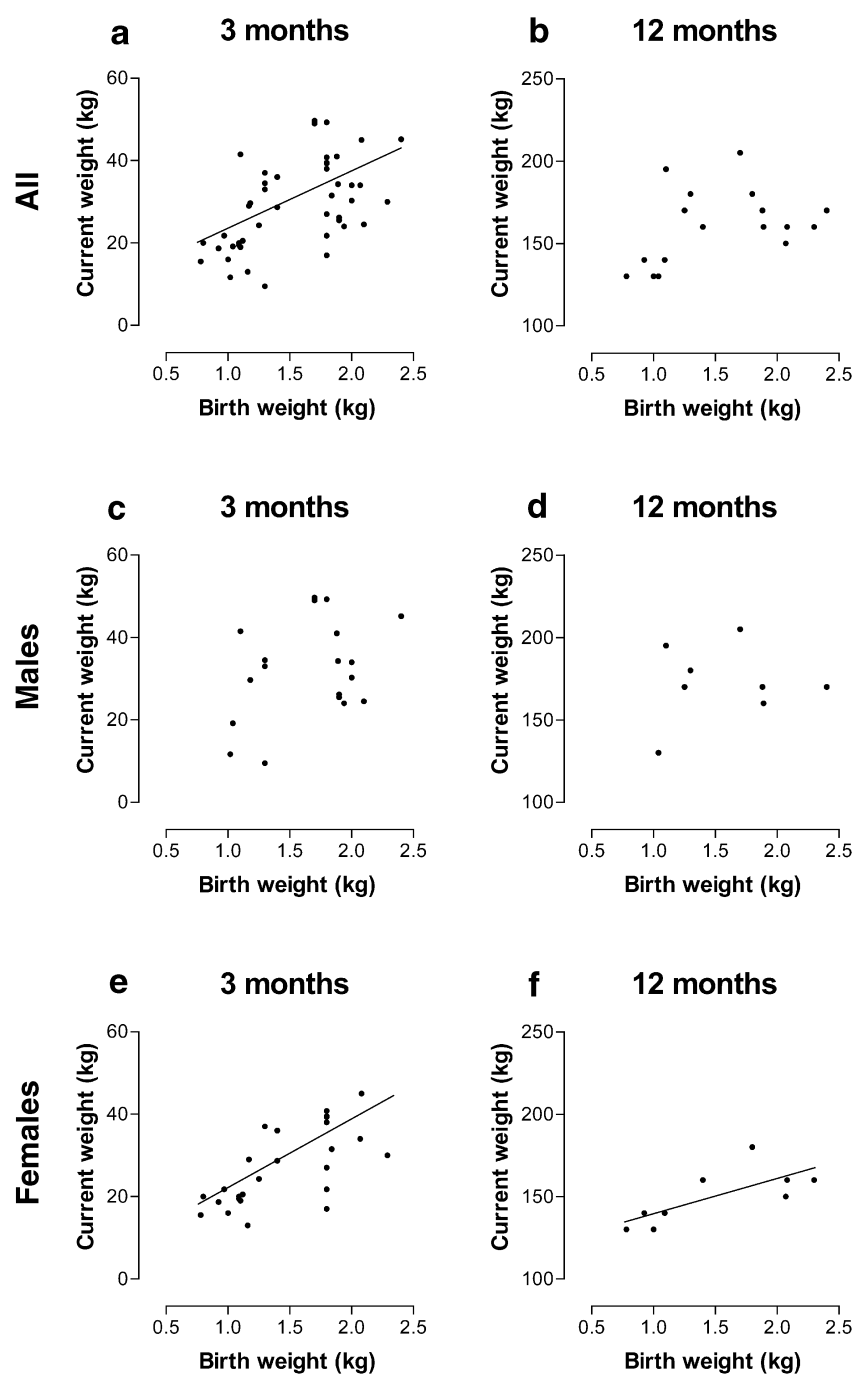

Fig. 1a-f. The relationships between (current) body weight and age and birthweight (BW) in the pigs studied. Body weight at 3 months (a, c, e) and 12 months $(\mathbf{b}, \mathbf{d}, \mathbf{f})$ is shown for all pigs $(\mathbf{a}, \mathbf{b})$, male pigs $(\mathbf{c}, \mathbf{d})$ and female pigs $(\mathbf{e}, \mathbf{f})$ pigs. Weight (a) at 3 months $=14.4 * \mathrm{BW}+8.7 ; r=0.48, p<0.001$. Weight (e) at 3 months $=17.2 * \mathrm{BW}+4.9 ; r=0.53, p<0.005$. Weight (f) at 12 months $=21.0 * \mathrm{BW}+118.6 ; r=0.73, p<0.05$. Numbers per group at 3 months: low-BW males, $n=7$; high-BW males, $n=12$; lowBW females, $n=15$; high-BW females, $n=13$. Numbers per group at 12 months: low-BW males, $n=4$; high-BW males, $n=4$; low-BW females, $n=5$; high-BW females, $n=4$

(Fig. 1a,b). However, when the data for males and females were analysed separately, there were sex-specific relationships between birth and current weights. In males, there was no difference $(p>0.05)$ in current weight between low and high-birthweight pigs at 3 or 12 months of age (Table 1), and there were no significant $(p>0.05)$ relationships between current weight at either of the postnatal ages and birthweight (Fig. 1c,d). In females, current weight was significantly lower in low-birthweight than in high-birthweight pigs (Table 1) and current weight was correlated $(p<0.001)$ with birthweight at 3 and at 12 months of age (Fig. 1e,f). Overall, low birthweight caused a sig-

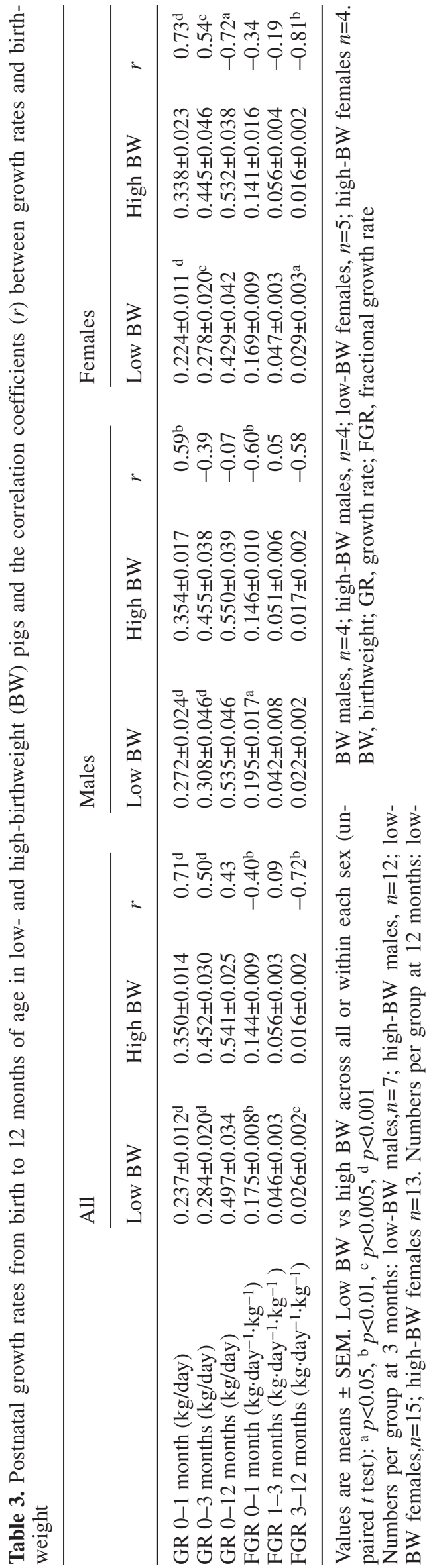


Table 4. Fasting glucose and insulin concentrations and glucose decrement after an insulin injection in low- and high-birthweight (BW) male and female pigs

\begin{tabular}{|c|c|c|c|c|c|c|}
\hline & \multicolumn{2}{|l|}{ All } & \multicolumn{2}{|l|}{ Males } & \multicolumn{2}{|l|}{ Females } \\
\hline & Low BW & High BW & Low BW & High BW & Low BW & High BW \\
\hline Fasting glucose (mmol/l) & $5.35 \pm 0.20$ & $5.43 \pm 0.20$ & $5.51 \pm 0.28$ & $5.54 \pm 0.26$ & $5.26 \pm 0.27$ & $5.28 \pm 0.33$ \\
\hline Fasting insulin (IU/ml) & $15.67 \pm 3.02$ & $15.79 \pm 2.01$ & $15.79 \pm 3.16$ & $15.41 \pm 2.30$ & $18.03 \pm 4.26$ & $16.3 \pm 3.71$ \\
\hline Glucose decrement $\left(\mathrm{mmol} \cdot \mathrm{l}^{-1} \cdot \mathrm{min}^{-1}\right)$ & $0.37 \pm 0.04$ & $0.33 \pm 0.02$ & $0.40 \pm 0.06$ & $0.32 \pm 0.03$ & $0.35 \pm 0.05$ & $0.34 \pm 0.04$ \\
\hline Fasting glucose (mmol/l) & $4.24 \pm 0.11$ & $4.81 \pm 0.15^{b}$ & $4.11 \pm 0.06^{\mathrm{a}}$ & $4.97 \pm 0.24$ & $4.34 \pm 0.20$ & $4.64 \pm 0.17$ \\
\hline Fasting insulin (IU/ml) & $10.49 \pm 1.47$ & $15.60 \pm 1.44$ & $8.27 \pm 1.35^{\mathrm{a}}$ & $15.20 \pm 1.62$ & $12.77 \pm 2.22$ & $16.00 \pm 2.64$ \\
\hline Glucose decrement $\left(\mathrm{mmol} \cdot \mathrm{l}^{-1} \cdot \mathrm{min}^{-1}\right)$ & $0.27 \pm 0.02$ & $0.32 \pm 0.03$ & $0.23 \pm 0.01$ & $0.33 \pm 0.03$ & $0.31 \pm 0.03$ & $0.32 \pm 0.03$ \\
\hline
\end{tabular}

Values are means \pm SEM. Low BW vs high BW across all or within

each sex (unpaired $t$ test): ${ }^{a} p<0.05,{ }^{\mathrm{b}} p<0.01$

Numbers per group at 3 months of age (fasting concentrations): low-BW males, $n=7$; high-BW males, $n=12$; low-BW females, $n=13$; high-BW females $n=9$. Numbres at 3 months (glucose decrement): low-BW males, $n=7$; high-BW males, $n=9$; low-BW females, $n=7$; high-BW females $n=5$. Numbers per group at 12 months: low-BW males, $n=4$; high-BW males, $n=4$; low-BW females, $n=5$; high- $\mathrm{BW}$ females $n=4$ nificant $(p<0.05)$ reduction in BMI and the ratios of head length to current weight and to $\mathrm{AC}$ at 3 but not at 12 months of age. However these effects were not statistically significant when males and females were analysed separately (Table 1 and Table 2). The head length to body weight ratio at 3 , but not 12 months of age, was positively related to that measured at birth overall $(r=0.45, n=47, p<0.05)$ and in females $(r=0.54$, $n=28, p<0.05)$. This was not the case with males $(r=0.60, n=19, p>0.05)$. Overall, the ratio of head length to AC was also positively related between birth and 3 months of age $(r=0.50, n=47, p<0.05)$, but this was not statistically significant within males and females when analysed separately.

Absolute postnatal growth rates (kg gained per day) from birth to 1 month and from birth to 3 months of age were smaller $(p<0.001)$ in low- than in high-birthweight pigs, an effect which was similar in males and females (Table 3). During suckling (birth to 1 month), the fractional growth rate ( $\mathrm{kg}$ gained per day, divided by starting weight in $\mathrm{kg}$ ) was greater $(p<0.001)$ in low than in high-birthweight pigs, yet when analysed in each sex, the fractional growth rate in this period was greater $(p<0.01)$ in low-birthweight male but not in low-birthweight female pigs (Table 3 ). During the post-weaning period of the study ( 1 to 3 months), the relative increase in body weight in low-birthweight pigs was not different to that in high-birthweight pigs in either sex (Table 3). Between 3 and 12 months of age, the fractional growth rate in low-birthweight pigs was greater $(p<0.005)$ than in high-birthweight pigs, however this was significant $(p<0.05)$ only in females (Table 3). Overall, birthweight significantly $(p<0.005)$ predicted growth rates between birth and 1 and 3 months and was negatively associated with the fractional growth rate during suckling (birth to 1 month) and between 3 and 12 months $(p<0.01$; Table 3$)$.
When analysed in males only, there was a positive relationship $(p<0.01)$ between birthweight and growth rate ( 0 to 1 month) and a negative relationship $(p<0.01)$ between birthweight and fractional growth rate between birth and 1 month (Table 3 ). In females only birthweight was positively related to growth rates at all stages of the study (birth to 1 month, $p<0.001$; birth to 3 months, $p<0.005$; birth to 12 months, $p<0.05)$ and negatively related to the fractional growth rate between 3 and 12 months $(p<0.01$; Table 3$)$.

Fasting glucose concentrations. At 3 months of age there was no difference between low- and high-birthweight pigs in either sex with regard to fasting glucose concentrations (Table 4). Overall, fasting glucose concentrations at 3 months of age were positively associated $(p<0.05)$ with current weight, growth rate (birth to 3 months) and fractional growth rate (1 to 3 months). They were negatively associated with the current head length to current weight ratio $(r=0.32$, $r=0.32, r=0.36$ and $r=-0.50$ respectively; $n=41)$. In males, but not in females, fasting glucose concentrations were significantly $(p<0.05)$ related to growth rate (birth to 3 months; $r=0.46, n=19$ ) and fractional growth rate (1 to 3 months; $r=0.54, n=19$ ). At 12 months of age, fasting glucose concentrations overall were lower in low-birthweight pigs than in high-birthweight pigs $(p<0.01$; Table 4$)$. When analysed within each sex, fasting glucose concentrations at this age were lower in low-birthweight male, but not in lowbirthweight female pigs $(p<0.05$; Table 4$)$, although glucose concentrations in 12-month-old males were not associated with any body weights, morphometric values or growth rates.

Fasting insulin concentrations. Overall, there was no difference in fasting insulin concentrations between 
low- and high-birthweight pigs at 3 or 12 months of age (Table 4). At 3 months of age, fasting insulin concentrations overall were negatively associated $(p<0.05)$ with the current head length to AC ratio $(r=-0.47, n=19)$. In males, but not in females, fasting insulin concentrations at 3 months were related $(p<0.05)$ to current weight, ponderal index, head length to $\mathrm{AC}$ ratio and growth rate from birth to 3 months $(r=0.45, r=0.64, r=-0.80$ and $r=0.56$ respectively; $n=19)$. At 12 months of age fasting insulin concentrations were lower $(p<0.05)$ in low-birthweight male pigs than in high-birthweight male pigs, but not in female pigs (Table 4). Overall, fasting insulin concentrations at this age were positively associated $(p<0.05)$ with weight, ponderal index and BMI at birth $(r=0.53, r=0.52$ and $r=0.67$ respectively; $n=17$ ), although analysis within each sex showed that only in males were insulin concentrations significantly related $(p<0.01)$ to birthweight and ponderal index at birth ( $r=0.84$ and $r=0.86$ respectively; $n=8$ ).

Hypoglycaemic response to insulin. At 3 months of age, there were no differences in plasma glucose concentrations during insulin challenge between lowand high-birthweight pigs, when considering all animals (Fig. 2a) or when analysed in males only (Fig. 2c) and in females only (Fig. 2e). At 12 months of age, the hypoglycaemic response to insulin was not different in low- and high-birthweight pigs overall (Fig. 2b). However, when analysed in each sex, plasma glucose concentrations were greater $(p<0.05)$ in low- than in high-birthweight female pigs (Fig. 2f) but not in male counterparts (Fig. 2d). There were no significant differences in the glucose decrement calculated during insulin challenge between low- and high-birthweight pigs at 3 or 12 months of age, or in male or female pigs when considered separately (Table 4). Overall, and in males and females separately, insulin sensitivity was not related to birthweight at either postnatal age. However, at 3 months of age, the glucose decrement was positively associated with the fractional growth rate between birth and 1 month of age $(p<0.01$, Fig. 3a) and with the head length to crown-rump length ratio at birth $(p<0.05, r=-0.39, n=28)$. When analysed in each sex, there were significant relationships $(p<0.05)$ in males between the glucose decrement at 3 months of age and the head length to AC ratio at birth $(r=0.55$, $n=16$ ) and the fractional growth rate in the first postnatal month (Fig. 3c). This did not apply in female pigs (Fig. 3e). In 3-month-old females, the glucose decrement was negatively associated $(p<0.005)$ with disproportionate current body shape (head length to crown-rump length ratio and head length to AC ratio; $r=-0.93$ and $r=-0.96$ respectively; $n=12$ ). By 12 months of age, the relationship between insulin sensitivity and the fractional growth rate between birth and 1 month of age switched to a
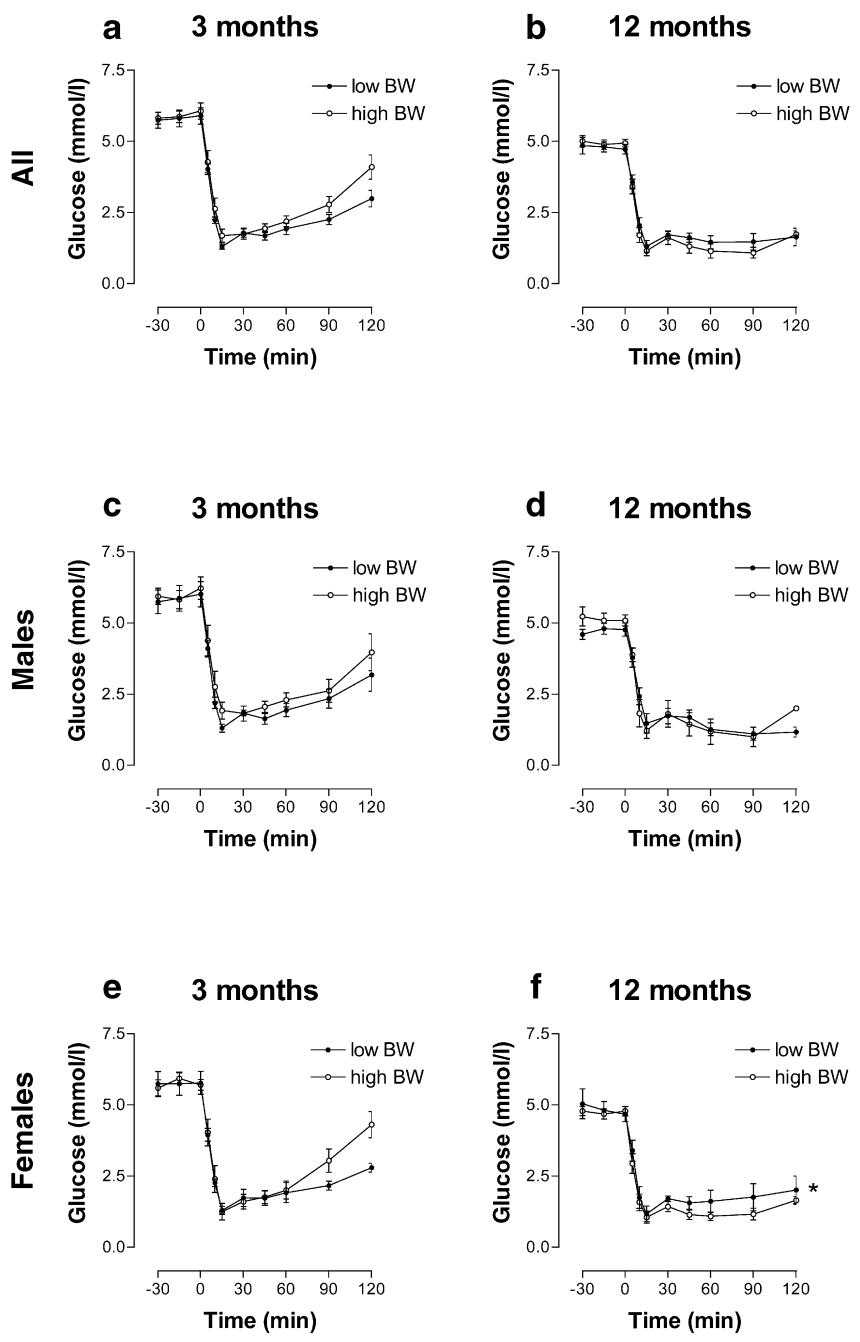

Fig. 2a-f. Plasma glucose concentrations in low- (O) and high- $(\bigcirc)$ birthweight $(\mathrm{BW})$ pigs after an i.v. insulin injection. The injection $(0.5 \mathrm{IU} / \mathrm{kg}$ body weight) was given at $0 \mathrm{~min}$ (horizontal axis). Glucose concentrations are for 3 months of age $(\mathbf{a}, \mathbf{c}, \mathbf{e})$ and 12 months of age $(\mathbf{b}, \mathbf{d}, \mathbf{f})$ in all pigs $(\mathbf{a}, \mathbf{b})$, in male pigs $(\mathbf{c}, \mathbf{d})$ and in female pigs $(\mathbf{e}, \mathbf{f})$. For each figure plasma glucose concentrations were reduced $(p<0.001)$ after insulin administration. During insulin challenge plasma glucose concentrations were greater $(* p<0.05)$ in low than in high-BW pigs in 12-month-old females only (f) (see*). There were no differences between low and high-BW pigs at 3 or 12 months of age in the minimum glucose concentration achieved after the insulin injection, either in absolute terms or when expressed relative to pre-experiment concentrations. At 3 but not 12 months of age absolute and relative glucose concentrations $120 \mathrm{~min}$ after the insulin injection were lower in low-BW than in high-BW pigs when groups were combined (a), and also in females alone (e). Numbers per group at 3 months: low-BW males, $n=7$; high-BW males, $n=9$; low-BW females, $n=7$; highBW females, $n=5$. Numbers per group at 12 months: low-BW males, $n=4$; high-BW males, $n=4$; low-BW females, $n=5$; highBW females, $n=4$

negative association (Fig. 3b). This relationship was similar in males and females, although it was not quite statistically significant $(p=0.07)$ in either group (Fig. 3d,f). 

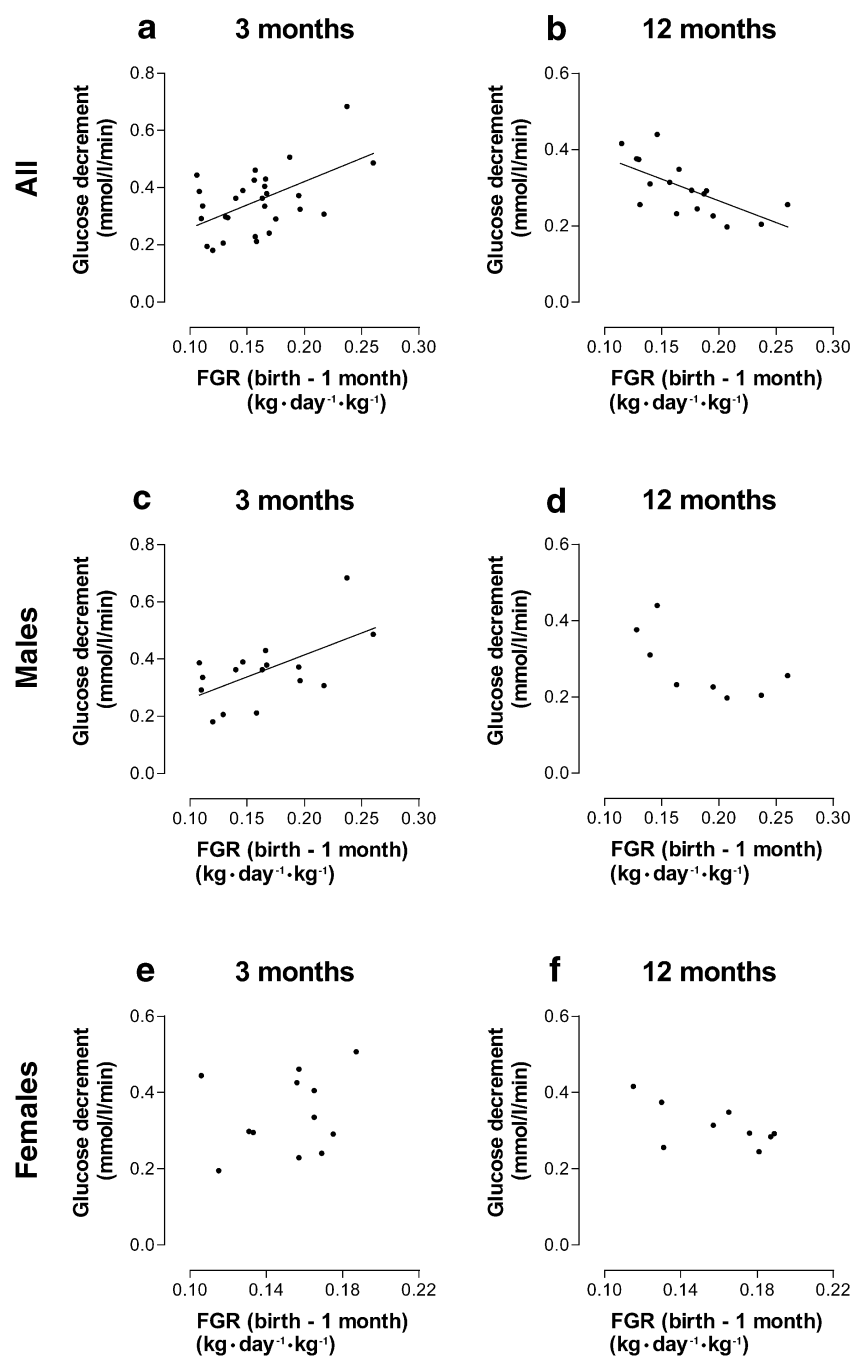

Fig. 3a-f. The relationships between fractional growth rate (FGR) from birth to 1 month and the glucose decrement after an insulin injection. Results are shown for 3 months of age (a, $\mathbf{c}, \mathbf{e})$ and 12 months of age $(\mathbf{b}, \mathbf{d}, \mathbf{f})$ in all pigs $(\mathbf{a}, \mathbf{b})$, in male pigs $(\mathbf{c}, \mathbf{d})$ and in female pigs $(\mathbf{e}, \mathbf{f})$. Glucose decrement (a) at 3 months $=1.42 * \mathrm{FGR}+0.13 ; r=0.50, p<0.01$. Glucose decrement (b) at 12 months $=0.50-1.26 * \mathrm{FGR} ; r=0.69, p<0.005$. Glucose decrement $(\mathbf{c})$ at 3 months $=1.53 * \mathrm{FGR}+0.11 ; r=0.59, p<0.05$. Numbers per group at 3 months: low-birthweight (BW) males, $n=7$; high-BW males, $n=9$; low-BW females, $n=7$; high-BW females, $n=5$. Numbers per group at 12 months: low-BW males, $n=4$; high-BW males, $n=4$; low-BW females, $n=5$; highBW females, $n=4$

\section{Discussion}

Our study shows that natural variation in intrauterine growth seen within litters of pigs affects postnatal insulin sensitivity. The relationship between intrauterine growth and postnatal insulin sensitivity was complex and was determined partially by the growth rate immediately after birth and partially by the sex and postnatal age of the pig. Birthweight was not directly related to postnatal insulin sensitivity but was an important factor in determining the growth rate of the piglets after birth. At 3 months of age, piglets with higher fractional growth rate during suckling were more insulin sensitive, whereas at 12 months of age these piglets were less insulin sensitive. These findings are consistent with human epidemiological observations and with previous studies on experimental animals, which have shown adult insulin resistance after impaired intrauterine growth [3, 7, 14]. Our results also indicate that the poor glucose tolerance seen previously in adult pigs of low birthweight [9] is more likely to be due to reduced insulin sensitivity than to insulin deficiency.

In pigs, the pattern of postnatal growth is highly dependent on birthweight, although the specific effects of low birthweight also depend on the sex of the piglet. In males, low birthweight and disproportionate body shape at birth is followed by a period of catch-up growth in the first month of life, as indicated by a high fractional growth rate. Although this high fractional growth rate was not maintained after weaning, by 3 months of age there were no longer any differences between low- and high-birthweight male pigs in body weights or proportions. Current weight was therefore unrelated to birthweight in male pigs at either 3 or 12 months of age. In contrast, in low-birthweight female pigs, postnatal growth rates in the first 3 months of life were lower than in their high-birthweight littermates and body weights of the low-birthweight group remained lower throughout the 12month study. Current weight was therefore directly related to birthweight in female pigs at 3 and at 12 months of age. In contrast to males, low-birthweight thin female pigs remained light at 3 months of age, but appeared to be relatively fatter at 12 months of age, as indicated by a lower ratio of $\mathrm{AC}$ to head length. Low-birthweight male pigs therefore had a very early phase of catch-up growth, which appears to be complete by puberty, whereas low-birthweight female pigs had less catch-up growth before weaning, but a high fractional growth rate during puberty.

The relationship between fractional growth rate during the first month of life and insulin sensitivity, measured as the glucose decrement in response to exogenous insulin administration, reversed between 3 and 12 months of age. A similar switch from improved glucose handling to glucose intolerance with increasing postnatal age has been observed in other species following intrauterine compromise [15]. In the current study, the timing of this switch differed in males and females and could be due to the sex-specific effects of low birthweight on postnatal growth patterns in pigs. Thinness at birth and subsequent catchup growth were associated with increased insulin sensitivity in male but not female pigs at 3 months of age. Low birthweight in females was not as closely related to increased fractional growth rate immediately after birth as in males and, hence low-birthweight females remained small at 3 months of age. Indeed, females 
that were disproportionate in body shape at 3 months of age were less insulin sensitive. By 12 months of age, higher fractional growth rates during suckling were associated with reduced insulin sensitivity in both male and female pigs. Thus, insulin resistance appears to develop between 3 and 12 months of age in low-birthweight male pigs with early catch-up growth, but could already be present at 3 months of age in low-birthweight female pigs which remained disproportionate in body shape. Certainly, the inverse correlation between fractional growth rate and insulin sensitivity in adult pigs is consistent with a previous finding that high fractional growth rate is associated with another index of insulin resistance, namely an increased ratio of insulin area under the curve to glucose area under the curve during the glucose tolerance test (unpublished observations by the authors).

Although the results from this and other studies suggest that the poor glucose tolerance of adult lowbirthweight pigs is due to reduced insulin sensitivity, there was no evidence of hyperinsulinaemia or hyperglycaemia in the fasted state, factors normally indicative of insulin resistance. In fact, in low-birthweight males, fasting insulin and glucose concentrations were lower at 12 months of age. Similar observations have been made in the offspring of sows which were growth retarded in utero due to maternal protein restriction during pregnancy [16]. Thinness and disproportionate shape at birth are associated with lower hepatic activity of the rate-limiting gluconeogenic enzyme, phosphoenolpyruvate carboxykinase, at 12 months of age [9]. Hence, the low glucose concentrations in low-birthweight male pigs could simply reflect a reduced capacity for glucose production during overnight fasting at this age, an effect possibly compounded by altered hepatic glycogen content, although we have no evidence for this. As insulin secretion in response to glucose appears to be unaffected by birthweight [9], the low basal insulin concentrations in male low-birthweight pigs could simply reflect low basal glucose concentrations. Alternatively, insulin clearance could be affected by low birthweight, thereby changing the basal glucose set-point. However, at 12 months of age, insulin, but not glucose concentrations were directly associated with low birthweight and thinness at birth in males, which suggests that low birthweight affects insulin concentrations independently of low glucose concentrations. In females, the absence of any effect of birthweight on fasting insulin and glucose concentrations at 12 months of age could reflect the increased ability of low-birthweight female pigs to maintain blood concentrations due to their increased accumulation of fat.

Together, the results of this study suggest that postnatal growth is a more important determinant of adult insulin sensitivity than low birthweight as such. In particular, catch-up growth in the first month of life in low-birthweight pigs was the best predictor of adult insulin resistance. In human cohorts, low birthweight coupled with rapid postnatal catch-up growth is also linked to insulin resistance and glucose intolerance during childhood and old age $[17,18,19,20]$. The relationship between catch-up growth and insulin sensitivity in our study was age-dependent. Since lowbirthweight female pigs did not show as marked an early catch-up growth, the high fractional growth rate of the low-birthweight males could be the consequence, rather than the cause of the increased insulin sensitivity. In conclusion, the pig is a useful model for investigating the intrauterine origins of abnormal glucose metabolism in adult humans and, in particular, how progression to adult onset degenerative diseases can differ in men and women.

Acknowledgements. This work was supported by The Wellcome Trust. We are grateful to P. Hughes for surgical assistance and S. Nichols and V. Johnson for care of the animals.

\section{References}

1. Barker DJ, Osmond C, Golding J, Kuh D, Wadsworth ME (1989) Growth in utero, blood pressure in childhood and adult life, and mortality from cardiovascular disease. BMJ 298:564-567

2. Hales CN, Barker DJ, Clark PM et al. (1991) Fetal and infant growth and impaired glucose tolerance at age 64. BMJ 303:1019-1022

3. Phillips DI, Barker DJ, Hales CN, Hirst S, Osmond C (1994) Thinness at birth and insulin resistance in adult life. Diabetologia 37:150-154

4. Hales CN, Barker DJ (1992) Type 2 (non-insulin-dependent) diabetes mellitus: the thrifty phenotype hypothesis. Diabetologia 35:595-601

5. Ozanne SE, Wang CL, Coleman N, Smith GD (1996) Altered muscle insulin sensitivity in the male offspring of protein-malnourished rats. Am J Physiol 271:E1128-E1134

6. Persson E, Jansson T (1992) Low birth weight is associated with elevated adult blood pressure in the chronically catheterized guinea-pig. Acta Physiol Scand 145:195-196

7. Moss TJ, Sloboda DM, Gurrin LC, Harding R, Challis JR, Newnham JP (2001) Programming effects in sheep of prenatal growth restriction and glucocorticoid exposure. Am J Physiol Regul Integr Comp Physiol 281:R960-R970

8. Petry CJ, Dorling MW, Pawlak D, Ozanne SE, Hales CN (2001) Diabetes in old male offspring of rat dams fed a reduced protein diet. Int J Exp Diabetes Res 2:139-143

9. Poore KR, Fowden AL (2002) The effect of birth weight on glucose tolerance in pigs at 3 and 12 months of age. Diabetologia 45:1247-1254

10. Bauer R, Walter B, Hoppe A et al. (1998) Body weight distribution and organ size in newborn swine (sus scrofa domestica) - a study describing an animal model for asymmetrical intrauterine growth retardation. Exp Toxicol Pathol 50:59-65

11. Poore KR, Forhead AJ, Gardner GS, Giussani DA, Fowden AL (2002) The effects of birth weight on basal cardiovascular function in pigs at 3 months of age. J Physiol 539: 969-978

12. Poore KR, Fowden AL (2002) Insulin sensitivity, plasma leptin and body fat in 3- and 12-month old pigs of varying birth weight. (Abstract) J Physiol 539:118P 
13. Agricultural and Food Research Council. Technical committee on response to nutrients (1990) Report No. 4, Nutrient Requirements of Sows and Boars. Nutr Abstr Rev Series B: Livestock Feed 60:383-406

14. Hales CN, Desai M, Ozanne SE, Crowther NJ (1996) Fishing in the stream of diabetes: from measuring insulin to the control of fetal organogenesis. Biochem Soc Trans 24:341-350

15. Ozanne SE, Hales CN (1999) The long-term consequences of intra-uterine protein malnutrition for glucose metabolism. Proc Nutr Soc 58:615-619

16. Atinmo T, Baldijao C, Pond WG, Barnes RH (1976) Maternal protein malnutrition during gestation alone and its effects on plasma insulin levels of the pregnant pig, its fetuses and the developing offspring. J Nutr 106:1647-1653
17. Crowther NJ, Cameron N, Trusler J, Gray IP (1998) Association between poor glucose tolerance and rapid post natal weight gain in seven-year-old children. Diabetologia 41:1163-1167

18. Bavdekar A, Yajnik CS, Fall CH et al. (1999) Insulin resistance syndrome in 8-year-old Indian children: small at birth, big at 8 years, or both? Diabetes 48:2422-2429

19. Eriksson JG, Forsen T, Tuomilehto J, Jaddoe V, Osmond C, Barker DJ (2002) Effects of size at birth and childhood growth on the insulin resistance syndrome in elderly individuals. Diabetologia 45:342-348

20. Forsen T, Eriksson J, Tuomilehto J, Reunanen A, Osmond C, Barker D (2000) The fetal and childhood growth of persons who develop type 2 diabetes. Ann Intern Med 133: 176-182 